UPIA, Rheumatoid Arthritis (RA), Spondyloarthritis $(\mathrm{SpA})$ or Psoriatic Arthritis (PsA), respectively.

Results: During the follow-up 6 (14.3\%) UPIA reached a defined diagnosis (2 RA, $2 \mathrm{SpA}$ and 2 PsA, respectively). At baseline, UPIA who differentiated had higher GSUS $(p=0.01)$ and PDUS scores $(p=0.02)$ compared to patients who remained as UPIA within 1 year. At baseline, UPIA who differentiated towards defined arthritis had higher histological scores for lining and sublining $\operatorname{CD}^{+} 8^{+}(p=0.005$ and $p=0.04$ for lining and sublining, respectively), sublining $C D 3^{+}$cells $(p=0.002)$ and $C D 31^{+}$vessels count $(p<0.001)$ than patients who remained as UPIA. In addition, there were direct correlations between baseline GSUS and PDUS scores with lining $C D 68^{+}$cells scores $(p<0.001$ for GSUS and $p=0.02$ for PDUS scores respectively), sublining $C D 68^{+}$cells scores ( $p=0.02$ for GSUS and $p=0.03$ for PDUS scores respectively), sublining $\mathrm{CD}^{+}$cells score $(\mathrm{p}=0.002$ for GSUS and $p=0.002$ for PDUS scores respectively) and CD $31^{+}$vessels count $(p<0.001$ for GSUS and $\mathrm{p}=0.01$ for PDUS scores respectively) in UPIA. Finally, the areas under the receiver operating characteristic (ROC) curves $C D 31^{+}$vessels count (cut-off value: 24.3), GS score (cut-off value: 1.5) and PDUS score (cut-off value: 1.5) were calculated to assess the best cut-off points to identify the differentiation likelihood during the follow-up in UPIA patients. The logistic regression analysis, demonstrated that having baseline GSUS and PDUS scores $\geq 1.5$ [OR:13.64 (95\% Cl: 0.98-242.59); $\mathrm{p}=0.05]$ and $\mathrm{CD} 31^{+}$vessels count $\geq 24.3$ [OR:51.13 (95\% Cl: 3.15-829.16); $p=0.01]$ were independent factors associated with the achievement of defined arthritis.

Conclusions: Histological and US assessment may help in the identification of patients with seronegative UPIA with high likelihood of clinical differentiation towards defined arthritis.

Disclosure of Interest: None declared

DOI: 10.1136/annrheumdis-2017-eular.4841

\section{SAT0067 FURTHER TREATMENT INTENSIFICATIONS IN UNDIFFERENTIATED AND RHEUMATOID ARTHRITIS PATIENTS ALREADY IN LOW DISEASE ACTIVITY HAVE LIMITED BENEFIT TOWARDS PHYSICAL FUNCTIONING}

S.A. Bergstra ${ }^{1}$, O. Olivas ${ }^{2}$, G. Akdemir ${ }^{1}$, N. Riyazi ${ }^{3}$, G. Collee ${ }^{3}$, J.H. Van Groenendael $^{4}$, R.B. Landewé ${ }^{5,6}$, C.F. Allaart ${ }^{1} .{ }^{1}$ Rheumatology, LUMC, Leiden, Netherlands; ${ }^{2}$ Rheumatology, Hospital Universitario 12 de Octubre, Madrid, Spain; ${ }^{3}$ Medical Center Haaglanden, The Hague; ${ }^{4}$ Reumazorg Southwest Netherlands, Bergen op Zoom; ${ }^{5}$ Amsterdam Rheumatology \& Immunology Center, Amsterdam; ${ }^{6}$ Zuyderland Medical Center, Heerlen, Netherlands

Background: It is recommended to optimize treatment as long as a predefined treatment target is not met, but should we aim at remission if patients are in low disease activity (LDA)?

Objectives: To assess if RA or undifferentiated arthritis (UA) patients who achieved LDA benefit with better functional ability from treatment intensification aimed at DAS remission.

Methods: In the IMPROVED study 610 patients with early RA (ACR 2010) or UA were "treated to target" aimed at DAS remission, assessed 4-monthly. Initial treatment was methotrexate (MTX) + tapered high dose prednisone. Patients with DAS $\leq 1.6$ tapered treatment. Patients with DAS $>1.6$ were randomized to MTX + hydroxychloroquine + sulphasalazine + prednisone or to MTX + adalimumab. Over 5 years, patients with DAS $>1.6$ were required to increase, change or restart medication. HAQ was measured 4-monthly. A linear mixed model analysis with random intercept was performed to test the relationship between changes in therapy and HAQ over time. Patients in LDA with DAS $>1.6$ with and without (i.e. protocol violation) treatment change were compared. $\triangle \mathrm{HAQ}$ and $\triangle \mathrm{DAS}$ at each visit compared to the previous visit were calculated. We tested the interaction effect between change in treatment and follow-up time adjusted for possible confounders.

Results: Overall, over 5 years DAS (baseline mean (SD) 3.2 (1.7)) and HAQ (1.2 (0.7)) showed a statistically significant and clinically relevant decrease $(\triangle \mathrm{HAQ}$ $-0.59,95 \% \mathrm{Cl}-0.61,-0.57 ; \Delta \mathrm{DAS}-1.77,95 \% \mathrm{Cl}-1.79 ;-1.75)$. The number of patients in LDA per visit ranged from 88 to 146 , of which $26 \%$ to $73 \%$ (increasing over time) had no treatment change due to protocol violations. We found a statistically significant but not clinically relevant effect of treatment change on $\triangle \mathrm{HAQ}$, corrected for baseline $\mathrm{HAQ}$, age, gender and treatment arm (model 1 , $\beta-0.085,95 \% \mathrm{Cl}-0.13,-0.044$ ). When $\triangle \mathrm{DAS}$ was added (model 2), the effect of treatment change was partly explained by $\triangle \mathrm{DAS}$ and no longer statistically significant $(\beta-0.022,95 \% \mathrm{Cl}-0.060 ; 0.015)$. The effect of treatment intensification on HAQ improvement became less over time, as demonstrated by a statistically significant interaction between change in HAQ and time in follow-up in model 3 ( $\beta$ $0.0098,95 \% \mathrm{Cl} 0.0010 ; 0.019$ ) (table 1).

Conclusions: Treatment intensification in early RA or UA patients who have already achieved low disease activity is associated with a statistically significant decrease in HAQ, but not with a clinically meaningful improvement in functional ability. The effect on $\triangle \mathrm{HAQ}$ decreased with increasing follow-up time. Therefore not remission or low disease activity, but good functional ability may be the optimal treatment target at which to steer treatment adjustments. These results suggest that, whereas remission may be the optimal goal, when patients in low disease activity have acceptably low $\mathrm{HAQ}$, further treatment intensification may only have downsides such as side effects and costs.

Disclosure of Interest: None declared
Table 1: Linear Mixed Model analysis to assess the effect of treatment intensification on change in $\mathrm{HAQ}$.

\begin{tabular}{|c|c|c|c|}
\hline & $\beta$ & $\mathbf{P}$ & $95 \% \mathrm{~d}$ \\
\hline \multicolumn{4}{|l|}{ Model $1(n \text { patients }=479, n \text { visits }=1528)^{\circ}$} \\
\hline Treatment intensification & -0.085 & $<0 . \infty 1$ & $-0.13 ;-0.044$ \\
\hline Follow-up timet & 0.0057 & 0.019 & $0.00094 ; 0.010$ \\
\hline \multicolumn{4}{|l|}{ Model $2(n \text { patients }=476, n \text { visits }=1509)^{*}$} \\
\hline Treatment intensification & -0.022 & 0.246 & $-0.060 ; 0.016$ \\
\hline Follow-up time $e^{b}$ & 0.0022 & 0.313 & $-0.0021 ; 0.0066$ \\
\hline DAS change & 0.23 & $<0 . \infty 1$ & $0.21 ; 0.26$ \\
\hline \multicolumn{4}{|l|}{ Model 3 (n patients $=476, n$ visits $=1509)^{\circ}$} \\
\hline Treatment intensification & -0.10 & 0.013 & $-0.18 ;-0.021$ \\
\hline Follow-up time & -0.0034 & 0.323 & $-0.010 ; 0.0033$ \\
\hline Treatment intensification " follow-up time & 0.0098 & 0.029 & $0.0010 ; 0.019$ \\
\hline DAS change & 0.23 & $<0 . \infty 1$ & $0.21 ; 0.26$ \\
\hline
\end{tabular}

$\mathrm{HAQ}=$ health assessment questionnaire, $\mathrm{SE}=$ standard error, $\mathrm{Cl}=$ confidence interval.

-All models were adjusted for age, gender, treatment arm and baseline HAQ.

"Follow-up time is added to the model as visit number, with time between visits being

4 months

DOI: 10.1136/annrheumdis-2017-eular.1606

\section{SAT0068 THE TIME UNTIL PERFORMING TIGHT CONTROL AS A TREAT-TO-TARGET STRATEGY AND THE TOLERABILITY OF METHOTREXATE STRONGLY INFLUENCE THE ACHIEVEMENT OF CLINICAL REMISSION IN RHEUMATOID ARTHRITIS}

T. Hagiwara, K. Kamada, N. Namura. Rheumatology, Takarazuka City Hospital, Takarazuka, Japan

Background: Clinical remission (CR) is the first targeted outcome of early treatment for rheumatoid arthritis (RA). Therefore, a consensus is needed for achieving CR by using the treat-to-target (T2T) strategy in RA treatment. However, in patients who received long-term insufficient treatment for RA, achievement of CR becomes increasingly difficult, especially if there is delay in the treatment.

Objectives: We aimed to examine factors that hinder successful RA treatment. We believe that making primary-care physicians aware of treatment results will increase the remission rate of RA.

Methods: We examined 388 patients with RA who were observed between January and October 2016 and who had not received new disease-modifying anti-rheumatic drugs (DMARDs) more than 3 months before the observation day. We investigated their age at RA onset, sex, Steinbrocker radiographic stage and functional class, activity level, rheumatoid factor (RF), the anti-cyclic citrullinated peptide antibody and DMARDs prescribed at the first consultation (Prescribed Before), disease activity, status of methotrexate (MTX), glucocorticoids (GCs), and biologic agent use at the last observational day.

First, we analysed the assumed remissions by using the Boolean-based definition (Boolean remission) as a purpose variable for these factors. Furthermore, we examined the odds ratio (OR) and $95 \%$ confidence interval $(95 \% \mathrm{Cl}$ ) by using a multiple logistic regression analysis for the statistically significantly different factors.

As for the time-related factor, we recognized that each factor had distinct multiplex collinear characteristics. Therefore, we adopted the time required for the first consultation as disease duration with the most effective values as the analysis object. The representative factor for the functional assessment adopted class according to the number of effective analyses.

Results: We recognised the statistically significant differences in disease duration, stage, class at the time of the first medical examination, RF, Prescribed Before, and state of MTX and GCs use at the last observation day for the achievement of Boolean remission.

We examined the multiple logistic regression analysis with the previously mentioned results and obtained the following results.

- Disease duration (per 1 year); OR 1.110, 95\% Cl 1.048-1.175, $p<0.001$.

- MTX (state; using vs no using); OR 2.522, 95\% Cl 1.560-4.076, p $<0.001$

- Class at 1st interview; OR 1.512, 95\% Cl 1.126-2.029, $\mathrm{p}<0.01$

- GCs (state; no using vs using); OR 1.803, 95\% Cl 0.912-3.565, p=0.090.

- Disease duration ( $<1.605 y$ vs $>1.605 y)$; OR $2.233,95 \% \mathrm{Cl} 1.437-3.470$, $\mathrm{p}<0.001$.

- MTX (state; using vs no using); OR 2.656, 95\% CI 1.644-4.291, p $<0.001$

- Class at 1st interview; OR 1.589, 95\% Cl 1.181-2.136, $\mathrm{p}<0.01$.

- GCs (state; no using vs using); OR 1.883, 95\% Cl 0.956-3.711, p=0.067.

Conclusions: Our results indicated the importance of the time required for consultation facilities with the T2T strategy treatment, tolerability for MTX use, and mild dysfunction at the first interview.

The window of opportunity to achieve remission for patients with RA has less time than expected. Therefore, we recommend that physicians should introduce patients with RA to a rheumatologist following the T2T strategy promptly when the primary care provided by the family physician is insufficient. 